\title{
Axel HONNETH, Ce que social veut dire
}

Aude Malkoun-Henrion

\section{OpenEdition}

\section{Journals}

Édition électronique

URL : http://journals.openedition.org/ress/3698

DOI : $10.4000 /$ ress.3698

ISBN : $1663-4446$

ISSN : 1663-4446

Éditeur

Librairie Droz

Édition imprimée

Date de publication : 15 mai 2017

Pagination : 267-272

ISSN : 0048-8046

Référence électronique

Aude Malkoun-Henrion, «Axel HONNETH, Ce que social veut dire », Revue européenne des sciences sociales [En ligne], 55-1 | 2017, mis en ligne le 23 novembre 2016, consulté le 19 janvier 2021. URL: http://journals.openedition.org/ress/3698; DOI : https://doi.org/10.4000/ress.3698

Ce document a été généré automatiquement le 19 janvier 2021.

(C) Librairie Droz 


\title{
Axel HONNETH, Ce que social veut dire
}

\author{
Aude Malkoun-Henrion
}

\section{RÉFÉRENCE}

Axel HONNETH, 2013-2015, Ce que social veut dire, Paris, Gallimard, « NRF Essai », 2 t., 352 et $400 \mathrm{p}$.

1 Ouvrage en deux volumes d'œuvres écrites entre 1990-2010, Ce que social veut dire est une somme philosophique qui résume le chemin intellectuel parcouru par l'actuel directeur de l'Institut de recherche sociale à Francfort. Axel Honneth y défend l'idée que le social doit s'entendre essentiellement dans les termes d'une lutte pour la reconnaissance. À cette occasion, il ne s'agit pas seulement de faire (re)découvrir certains des écrits de l'auteur mais de donner à lire une pensée en acte. Le premier volume rassemble onze textes ayant pour objectif, via des «confrontations » avec des penseurs modernes et actuels, l'élaboration d'un « modèle du conflit social fondé sur la reconnaissance» (vol. I, p.19). Le second est composé de quatorze articles visant l'« élargissement normatif de la théorie de la reconnaissance » (vol. II, p. 18).

2 Intitulé «Le déchirement du social », le premier volume constitue une justification théorique de l'intuition de Honneth, celle de la dimension morale de tout conflit social. Honneth commence par distinguer deux lectures divergentes de l'origine des conflits sociaux. L'une, utilitariste, ramène ces derniers à n'être que l'expression d'intérêts individuels ou collectifs. L'autre, intersubjectiviste, présente deux versions d'une même prémisse hégélienne "selon laquelle le sujet, pour accéder à la conscience de soi ou jouer un rôle dans la société, a besoin d'une reconnaissance » (p.13) de la part des autres membres du groupe auquel il appartient. Honneth pose également en introduction une définition de la reconnaissance comme "jugement généralisé des autres membres sur la pertinence de [nos] accomplissements cognitifs ou pratiques » (p. 14). C'est donc à partir de la thèse de la reconnaissance qu'il construit son modèle du conflit social. En effet, ainsi comprise, la reconnaissance comporte nécessairement une part de lutte issue des désaccords qu'implique le choix des critères donnant lieu et 
validité à ces jugements. Cela établi, Honneth entreprend d'examiner les deux versions, allemande et française, de cette thèse intersubjectiviste fondée sur la reconnaissance mutuelle. Les quatre premiers chapitres présentent les racines allemandes de la notion. Le chapitre 1 aborde l'idée kantienne qu'une concurrence permanente pour l'estime sociale, dans un contexte socioculturel favorisant les conduites morales et l'usage public de la raison, peut constituer un moteur du progrès moral. Le chapitre 2 présente la version fichtéenne de la reconnaissance conçue comme l'appel réciproque d'un statut normatif, celui de sujet libre. Les deux suivants sont consacrés à sa lecture de Hegel. La reconnaissance, entendue ici comme acte réciproque d'autodétermination, est autant la condition de possibilité de la conscience de soi que celle préalable et «nécessaire à toute morale » (p. 104). Conçue comme le schème de «toutes les formes d'autolimitation " (p.18), elle se manifeste diversement et conflictuellement dans les « complexes institutionnels » de la famille, la société civile et l'État (p.126) et confère à la lutte, mue par le désir d'une intégration sociale toujours plus ample et effective, un caractère éthique.

3 L'idée d'une constitution socialement déterminée des agents et de leur liberté étant posée, Honneth s'intéresse ensuite (chapitres 5-10) à l'interprétation française de la thèse intersubjectiviste. Selon lui, sa principale caractéristique est de présenter une vue pessimiste des effets de ce rapport constitutif des individus. Vue pessimiste qu'il enracine dans la notion rousseauiste d'amour propre qui souligne l'essentielle et indépassable (inter)dépendance de l'individu à l'égard des autres. Chez Jean-Paul Sartre, Pierre Bourdieu, Luc Boltanski et Laurent Thévenot, par exemple, la quête de reconnaissance sociale échoue à étendre les libertés individuelles et conduit, en définitive, à reproduire des situations d'assujettissement. Cette tradition du soupçon naît d'un défaut de normativité du concept de reconnaissance qui, d'«action protomorale » chez Hegel, finit par désigner chez ces auteurs "plutôt une pratique cognitive » (p. 24) toujours vouée à l'échec. En d'autres termes, « une relation d'entente communicationnelle entre sujets n'est pas possible, puisque l'un des sujets doit toujours se trouver dans l'état objectivé de l'être-pour-autrui » (p. 137). Ce négativisme est autant un renversement qu'une réduction de perspective. Déjà, chez le jeune Sartre, l'objet étudié n'est plus la reconnaissance d'autrui mais celle de soi par autrui ; l'angle d'analyse adopté n'est plus l'acquisition par autolimitation réciproque d'une liberté toujours plus grande mais les effets possibles de réification mutuelle de sujets pris dans des rapports d'objectivation les limitant dans leur compréhension d'euxmêmes (p. 145).

4 Sa lecture de Claude Lévi-Strauss, Maurice Merleau-Ponty et Cornelius Castoriadis est en revanche moins marquée par le pessimisme de leur position que par l'importance des concepts-clés pouvant étayer sa conception du social. Des idées et concepts mis en avant par Lévi-Strauss, Honneth retient notamment le «sentiment de solidarité [...] envers l'environnement naturel » (p. 166) » qui caractérise la pensée « sauvage ».

Enfin, le dernier texte de ce recueil, «Sur la théorie de la justice de David Miller », se veut autant conclusif que transitif. Conclusif car il répond explicitement à une question de méthode évoquée précédemment, à savoir la place que les données empiriques peuvent et doivent occuper dans l'élaboration des théories sociales et politiques, par l'affirmation d'une «dépendance réciproque entre les sciences sociales et la philosophie politique»( . 278). Transitif car la problématique de la justification 
normative d'une diversité factuelle de conflits sociaux fait justement l'objet du recueil suivant.

6 Intitulé "Les pathologies de la raison », le second volume s'interroge sur le type de normativité qu'il s'agit d'accorder à la théorie de la reconnaissance. Deux dimensions peuvent être rattachées aux luttes pour la reconnaissance. L'une, éthique, répond au critère aristotélicien de la "réalisation d'une "vie bonne" ", l'autre, juridique, à celui d'une "répartition équitable des libertés individuelles» (p.12) au sein de la société. D'où le fait que les entraves à la réalisation de soi peuvent être qualifiées de pathologies sociales, alors que l'inégale répartition des droits positifs représente un état d'injustice. Ces valeurs normatives appartiennent à des champs philosophiques différents qu'il importe de distinguer. La première est la préoccupation de la philosophie sociale, alors que la seconde est celle de la philosophie politique. Conséquemment, si les luttes pour la reconnaissance sont à la fois les indices de pathologies et d'injustices sociales, il convient d'adapter le "modèle hégélien du conflit [à ces] deux langages théoriques " (p.15). Les articles regroupés ici répondent donc à deux objectifs : déterminer les conditions de possibilité d'un « diagnostic pathologique » et développer une notion de justice « adaptée au modèle de la lutte pour la reconnaissance » (p. 16). C'est au premier qu'est consacrée la première partie et au second que se rattache la troisième. La deuxième porte quant à elle sur les "conséquences de la psychanalyse " pour une théorie de la reconnaissance.

7 La première partie (chapitres 1-9) propose ainsi une relecture des écrits de la théorie critique à l'aune de la distinction établie en introduction entre philosophies sociale et politique. Honneth y range les différents travaux des membres de l'école de Francfort du côté de la problématique du diagnostic pathologique. Plus précisément, ce courant s'est attaché à formuler, dans les termes d'une théorie de la rationalité, les critères permettant l'accomplissement d'individus libres au sein des sociétés. De Theodor Adorno à Jürgen Habermas, il s'agit bien d'identifier « "l'universel rationnel" » (p. 17) que le système capitaliste mutile engendrant ainsi des pathologies et la souffrance des individus. Dans cette perspective, les chapitres 4 et 5 accordent respectivement un intérêt particulier à la notion de "perte d'expérience » (p. 104) et à la «critique de la violence " chez Benjamin. Quant au chapitre 3, il revient sur l'usage de la notion de critique au sein de la théorie critique et en propose un renouvellement. Approfondissant la distinction de Michaël Walzer des trois types de critique, Honneth montre que seule une critique réunissant " en un programme unique » (p. 94) les tâches des modèles constructif, reconstructif et généalogique est à même de questionner l'ordre social existant.

8 La deuxième partie (chapitre 10-12) cherche à mettre en évidence la valeur d'explanans de phénomènes concrets en lien avec la lutte pour la reconnaissance sociale dont font preuve les théories de l'attachement. L'intérêt de ces théories est de fournir une compréhension réaliste des forces psychiques qui poussent les individus et les groupes à exiger des formes nouvelles de reconnaissance. Honneth rappelle les trois sphères d'interactions sociales au sein desquelles existent trois modèles de reconnaissance (amour, droit, solidarité). Ces trois formes de reconnaissance mutuelle sont toutes nécessaires, à des niveaux différents, à l'acquisition de l'autonomie et à la réalisation de soi (confiance en soi, respect de soi, estime de soi). Ceci explique notre aspiration à la reconnaissance, au sein des groupes, de la valeur de nos personnalité et actions. Mais 
de tels groupes souffrent aussi de pathologies puisqu'ils sont à la merci d'événements régressifs qui menacent l'identité des individus.

9 La troisième et dernière partie (chapitres 13-14) retourne à l'objectif principal du livre, à savoir la mise au point d'un programme définitionnel du concept de justice dans le cadre de la théorie de la reconnaissance. Les théories de la justice dominantes, procéduralistes, s'accordent sur une conception strictement individuelle de l'autonomie et sa conséquence directe, « un paradigme de la distribution» (p. 295). Mais si la juste distribution des biens fondamentaux permet de réaliser la liberté des individus, c'est parce que ceux-ci sont déjà autonomes, à même de reconnaître des fins méritant d'être poursuivies et les capacités dont ils disposent pour leur réalisation. De même qu'une délibération citoyenne justifiant des principes de distribution nécessite des sujets libres et autonomes. Ici, l'autonomie est doublement présupposée. Or, celle-ci est « une grandeur relationnelle, intersubjective, pas un acquis monologique » (p. 302). Elle requiert la reconnaissance mutuelle des individus. Il en résulte qu'une théorie de la justice qui tienne compte des relations recognitives doit définir différemment son objet (principe matériel) et sa méthode (principe formel) : i.e. détrôner le principe suprême d'une justice distributive et "reconstruire" ses principes "à partir du processus historique des relations de reconnaissance " (p.313). Enfin, le recueil se termine sur une tentative d'application de la théorie de la reconnaissance à un autre type de relation, les relations interétatiques.

Le premier mérite de ces deux volumes est d'offrir une vue d'ensemble critique et progressive du cheminement de leur auteur. La division, proposée par l'éditeur et supervisée par Honneth, a cet effet positif et instructif de reproduire le mouvement d'une pensée en acte, de donner à voir ses questionnements et ses hésitations, ses découvertes et ses affinités intellectuelles et ce, sous un nouveau jour. La construction du premier volume, par exemple, met en évidence la double généalogie de sa théorie de la reconnaissance, les racines hégéliennes et « rousseauistes ", et éclairent ainsi le cœur théorique de l'une de ses œuvres majeures, La Lutte pour la reconnaissance. Alors que le second volume, quant à lui, s'apparente, par ses réflexions sur la puissance critique de la théorie de la reconnaissance, à la " fabrique » de son autre grande œuvre, Le Droit de la liberté. La problématique déployée, la constitution essentiellement conflictuelle et morale du social, confère une unité à des références à première vue disparates, à la fois philosophiques, sociologiques et anthropologiques. Et si le thème du conflit social n'est évidemment pas une «découverte personnelle» (vol. I, p. 9) de Honneth, son œuvre n'en demeure pas moins l'une des réactualisations contemporaines les plus importantes. C'est donc à la fois avec plaisir et profit que nous (re)lisons ces textes qui articulent brillamment analyses sociologiques et énoncés axiologiques. Ceci nous conduit à souligner le second intérêt majeur de cette publication: la distinction clairement établie entre philosophies sociale et politique. En effet, celle-ci aide à diviser les questionnements normatifs entre les conditions de la vie bonne et celles de la vie équitable et paisible. Elle permet aussi de résoudre l'une des ambigüités de la notion de reconnaissance, à savoir son caractère multidimensionnel. En outre, par cette différenciation des problématiques, Honneth inscrit sa réflexion dans la tradition de la théorie critique tout en lui conférant une place particulière : celle d'une troisième génération dont l'objectif est double en ce qu'elle vise à la fois « la justification d'un diagnostic pathologique » et « la légitimation de la notion de justice dans la perspective 
d'une théorie de la reconnaissance " (vol. II, p. 15). Saluons enfin son analyse de la notion de critique et le renouvellement qu'il en offre.

11 Venons-en à présent aux limites de ces deux volumes ou, plus justement, à nos « regrets » de lecteur. La première consiste en l'impasse faite, dans le premier volume, sur la réactualisation anglo-saxonne, pourtant incontournable, de ce thème hégélien. La «théorie néo-pragmatiste de la liberté » de Robert Pippin et Robert Brandom par exemple, définit la reconnaissance comme la confirmation, la validation, par autrui d'une prétention, ou revendication/exigence (claim), de teneur normative dans le cadre social de normes préétablies. Dans cette perspective, les demandes de reconnaissance s'élaborent au sein d'un espace de raisons (Sellars, 1956), c'est-à-dire, dans un contexte normatif commun, dans un jeu social de demandes/offres de justifications. Or, ici, Honneth déploie surtout la dimension juridique de la notion de reconnaissance. Ce qui nous conduit à la seconde limite de ces deux volumes : la préférence actuelle qu'il accorde à cette seule dimension. Préférence que Le Droit de la liberté, aboutissement des recueils, met explicitement en avant. Quoique la justification normative du modèle de la lutte pour la reconnaissance soit fondamentale, la question des causes de cette lutte et de son caractère inachevé demeure ouverte et essentielle à explorer. Non seulement pour la compréhension des phénomènes concrets de privation d'autonomie, mais aussi pour celle de ce qui nous définit en tant qu'humains, notre sociabilité. Certes, Honneth n'abandonne pas totalement le motif anthropologique comme en témoignent les articles recensés ici. Cependant, dans cette somme qui s'intitule Ce que social veut dire, l'attention prépondérante accordée à la dimension juridique de la reconnaissance nous paraît réduire le sens à accorder à la notion de « social » et, par là, affaiblir l'originalité qui jusqu'à lors caractérisait la position de Honneth parmi les théories contemporaines de la reconnaissance.

\section{AUTEURS}

\section{AUDE MALKOUN-HENRION}

Université de Paris 1-Sorbonne, NOSOPHI - Université de Montréal, CRE 